\title{
以助教的视角看定量分析化学实验的教与学
}

\author{
刘俊杰, 朱志伟 \\ 北京大学化学与分子工程学院，北京 100871
}

摘要: 定量分析化学实验教学的质量不仅关系到学生对于分析化学相关知识的理解程度, 同样也对学生的实验基本 操作训练影响深远。本文从一名兼具学生与教师双重身份的助教视角入手, 着重关注影响定量分析化学实验教学质 量的几大因素, 试图寻找提升教学质量的方案, 为广大定量分析化学实验的教学者提供参考。

关键词: 定量分析化学实验; 实验教学; 助教

中图分类号: G64; O6

\section{Teaching and Learning of Quantitative Analytical Chemistry Laboratory from the Perspective of Teaching Assistant}

\author{
Junjie Liu, Zhiwei Zhu * \\ College of Chemistry and Molecular Engineering, Peking University, Beijing 100871, China.
}

\begin{abstract}
The teaching quality of quantitative analytical chemistry laboratory course is not only related to students' understanding of analytical chemistry, but also has a profound impact on their basic laboratory skill training. In this paper, from the perspective of a teaching assistant who is both a student and a teacher, we focus on several factors that affect the teaching quality of quantitative analysis chemistry laboratory course, and try to find a plan to improve the teaching quality, so as to provide reference for quantitative analytical chemistry laboratory course teaching.
\end{abstract}

Key Words: Quantitative analytical chemistry laboratory; Laboratory teaching; Teaching assistant

\section{1 引言}

一门课程的教学效果很大程度上取决于任课教师的综合素质。多年来, 实验课助教一直是北京 大学化学与分子工程学院(简称 “学院” ) 定量分析化学实验教学的骨干力量, 他们的 “言” (对知识 点的讲解)与 “行” (实验技能的示范)直接影响到学生对实验课程的掌握程度。由于近年来分析化学 学科以及实验教学条件等都得到了突飞猛进的发展与改善, 从2018年春季学期开始, 学院对定量分 析化学实验的教学内容进行了大幅度的调整, 撤销 3 个容量滴定实验, 新增 1 个实际样品实验及 4 个仪 器分析实验 (电位分析、紫外光谱、红外光谱和荧光光谱) ${ }^{[1,2]}$ 。在以化学分析为主的课程中, 因为各 化学分析方法之间的系统性和连贯性, 助教对这些知识点及相关实验技能的掌握相对容易, 将他们 培训成能够胜任全部实验教学的 “全能型” 助教也不是很难。而在化学分析和仪器分析并重的课程 中, 由于仪器分析各方法之间相对独立, 每一种方法都涉及很多不同的新内容, 在短时间内要将一 个研二学生培训成 “全能型” 助教近乎不可能。因为再优秀的 “全能型” 助教也比不过 “摸爬滚 打” 多年的专职教师。因此, 学院对助教的培训策略由以往的 “大而全” 转变为 “专而精” 。培训 
教师会根据助教的研究专业方向以及他们在基础培训阶段的表现来指定各助教的具体实验教学内容 (即每位助教只负责 2-3个实验)。助教在 “吃透” 所分配实验的理论知识后, 在培训教师的指导下对 该实验反复做上4-5次, 认真发现、体会该实验的重点和难点, 尽可能模拟出学生在实验中可能会出 现的各种问题, 并与教师进行充分的讨论。助教通常能够在摸索、思考和讨论这样一种循环反复的 过程中自然而然地形成正确的解决方案。与此同时, 培训教师的言传身教也能在不经意间将有效的 教学方法和技能传授给助教。笔者就是通过这种方式培训出来的一位 “专业型” 助教, 负责 “经典 滴定与电位滴定” 和 “光度法” 这两个实验的教学工作, 授课人数共 208 人。经过近一个学期的教与 学, 尤其是从学生到教师的角色互换, 笔者对实验教学尤其是定量分析化学实验的教学有一些新的认 识, 希望这些想法和建议能为广大定量分析化学实验的教学者提供参考。

\section{2 实验教学目的及方向}

\section{1 加深知识理解, 提升动手能力}

理论知识的学习往往不容易被吸收, 而实践操作则可以让学生通过视觉、听觉等方面的刺激来 进行更为直观的学习, 感官可及的化学或物理变化也有助于形成深刻的印象, 这对于知识的接纳、 理解、吸收与转化都是极其有利的。作为实验性学科, 绝大部分成果都来源于实验结果, 实验操作 能力一定程度上决定了实验结果的准确性。优秀的实验操作能力包括规范的操作、合理的时间安排、 良好的应变能力等。学生动手能力的提高对其后续的实验、科研甚至生活具有重要意义。

\section{2 联系实际体系, 突出实用价值}

具备严密逻辑的理论知识终究要在实际生活生产中找到应用, 而实验则是二者的桥梁。除了加 深对于理论知识的理解, 实验教学同样承担着联系生活实际的作用 ${ }^{[3]}$ 。学院部分定量分析化学实验 的设计在一定程度上联系了实际应用体系, 如工业碳酸锂中微量铁的测定、未知酸分子量的测定等 均体现了定量分析的实际应用。理论联系基础实验、基础实验与实际体系并行能够更好地将知识连 贯起来, 从而获得更好的教学效果。

\section{3 内涵绿色化学, 培养良好习惯}

在助教培训以及后续的实验要求中, 更加注重绿色化学的理念, 如节约使用药品、限制铬酸洗 液的使用范围以及清洗铬酸洗液的水量不得超过 $80 \mathrm{~mL}$ 等。实验教学与生产生活应该一脉相承。资 源节约、环境保护等理念应该渗透到学习、科研、工作的各个方面。将绿色化学深入每个学生的内 心，久而久之，习惯也就成了自然。

\section{4 提升创新能力, 培养合作精神}

尽管大部分实验内容都建立在已有的实验教案之上, 但对于学生而言依然存在很大的思考空间。 通过对理论知识的 “再认识”, 他们的视野将更加开阔, 认知也更加完整, 具备更强的能力去创新 和创造。合作是当下科研以及工作中常用的一种提升效率、解决问题的手段。定量分析化学实验中 的小组实验是建立合作关系的一个平台, 通过合作能够让学生学会运用各种资源、互利共赢, 提升 工作效率。

\section{3 实验教学中的难点与思考}

\section{1 凡事预则立, 不预则废一一预习}

预习是知识学习过程中一个重要环节, 对于存在一定危险性的实验而言更是如此。良好的预习 有助于将理论知识与实验衔接起来, 不仅有利于实验的顺利进行, 也有利于学生加深对基础知识、实 验原理和基本操作等的理解。

实验教学过程中, 很多情况下学生的预习效果并不好, 课堂上抛出的一些问题并不能很快得到 学生的正确反馈。目前要求的预习报告作为相对有效的预习检查手段虽然能在很大程度上让学生熟 悉实验原理、步骤、数据处理等, 但依然存在相当大的局限性。主要表现如下: (1) 部分学生为了避 
免繁琐的预习报告占据自己的时间, 会提前将所有的预习报告写好, 最终导致的结果是实验前却忘 记了当前的实验内容; (2) 部分学生在写预习报告的过程中并不会深入思考实验原理、设计思路以 及操作重点等, 书面预习报告的形式使得预习实验演变成了抄写预习报告; (3) 脱离预习内容, 将预 习内容与实验操作割离开, 其显著特点就是淡化实验讲义中的部分内容而自行选择其实验方式, 如 有关移液管的操作, 讲义要求 “静置 $15 \mathrm{~s}$ 待溶液全部流下”, 实际操作却是 “移取完溶液后即拿开”。

在对 “光度法” 成绩(62份样本)进行数据统计时发现, 预习报告成绩与实验操作成绩正相关, 且 其相关系数达 0.70 , 由此表明实验操作质量与预习情况之间的强相互作用(图1)。但遗憾的是, 这种 正相关性并没有在 “经典滴定与电位滴定” 实验中得到很好的体现, 推测其原因可能与相对重复的 滴定操作有关。而在 “光度法” 实验中, 由于操作内容繁琐、操作步骤较多, 这种正相关性能够得 到更好的体现。当然这也从侧面反映出预习的重要性。

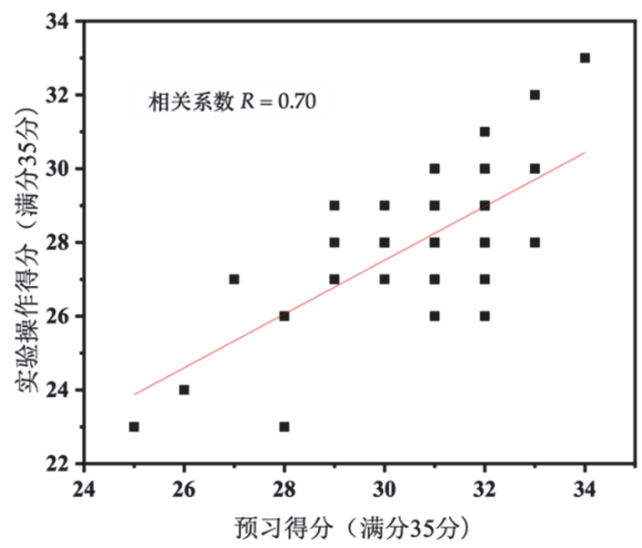

图1 实验操作与预习之间的相关性

就目前的教学情况来看, 笔者提出四点建议来提高学生的预习质量。(1) 大幅度提高预习报告 中归纳整理及思考的分值权重, 对以抄写实验讲义为主的预习报告则适当降低分数; (2) 在实验课 前1-3天提出若干问题, 要求学生将回答写入预习报告中; (3) 课前小测, 通过若干问题考查学生的 预习情况; (4) 课堂提问, 但由于课堂提问占据时间相对较多且效率较低, 因此还需要考虑更优的办 法来改进。

\section{2 知其然, 知其所以然一一思考}

基础学科中思维训练的一个要求便是透过现象察其本质, 现象具有多面性, 但本质却是统一的。 在以 “量” 为基本要求的定量分析化学实验中亦然。以对 “经典滴定与电位滴定” 实验中滴定突跃 的理解为例, 在经典滴定部分, 其本质在于溶液 $\mathrm{pH}$ 变化的连续性以及实验中能引起 $\mathrm{pH}$ “急剧” 变化 的量可以体现在一滴、半滴甚至更少的溶液体积中, 如此便很容易理解酸碱浓度、体积等对于突跃 的影响。而在电位滴定部分, 其本质在于玻璃膜电极表面的硅酸盐网络对氢离子的特异性响应而导 致的电位变化, 而深究这种响应则可以引发对于离子选择性电极的思考(如 $\mathrm{pH}$ 计的工作原理和适用 性); 关注氢离子扩散-吸附平衡则有助于快速理解摚拌、读数等操作的注意事项, 理解建立在电化学 势平衡基础之上的膜电位分析则可以很快获得电位- $\mathrm{pH}$ 响应关系。在教学过程中, 部分学生对于滴 定过程中电位-氢离子活度变化理解不够, 从而容易出现无法判断异常值、滴定过度等情况, 其根本 在于对基本原理理解得不够深入。

现象易得而本质难察, 这也是很基本的道理。如何在实验过程中教会学生深入理解是授课者值 得思考的问题, 毕竟实验教学很重要的一个目的就是加深对知识的理解。“溯源” 和归纳是两个主 要途径。仍以电位滴定为例, 观察到的现象是 $\mathrm{pH}$ H计上电位的变化, 而电位的变化在于电路输出中两 电极的变化, 建立在内参比电极电势稳定的认知上, 很容易想到是玻璃膜上电位的变化导致的结果, 
进一步溯源则可以建立氢离子活度与电位变化之间的关系……当溯源至硅酸盐网络对于氢离子特异 性响应时便足以理解涉及到电位滴定理论与实验中几乎所有的问题。当然, “溯源” 和归纳要建立 在足够的背景知识以及正确的思考方式基础上, 这就需要学生具备相应的知识与能力。

\section{3 进退有度, 过犹不及一有度}

定量分析化学实验以 “准确的量” 为目标, 在追求 “准确的量” 的过程中如何在每一步实验操 作中做到 “有度”也是实验教学的一个重点。

对 “准确的量” 的追求常常使得许多学生在实验操作中因担心产生误差而在每个步骤都谨小慎 微, 结果往往是适得其反: 其一是降低了工作效率, 浪费了时间; 其二是很难在较长时间内保持注 意力集中, 因此合理调配注意力显得更为重要; 其三是不利于实验过程中的及时思考。定量分析化 学实验中如何合理地、选择性地把握重点来进行精细化操作关系到实验的质量。实验过程中, 做 到 “有度” 至少应该做到三点: 其一, 关键步骤细节不可忽视(表1列出了一些常见的细节性错误), 如在电位滴定过程中将靠在烧杯内壁上的溶液清洗下去, 这一步操作尽管难度不大, 但关系到实验 的成败; 其二, “有度” , 合理地进行关键操作的细节处理而非追求所谓的 “极致” , 例如在电位 滴定实验中部分学生以 “获得更准确的实验结果” 为由在距离滴定终点几毫升便开始逐滴加入, 其 最终的结果是不仅难以获得更佳的实验结果, 而且极大地降低了实验效率。也有部分学生配制标准 溶液时为了减少烧杯中溶液的残留而过分洗涤烧杯; 其三, “粗细有度”, 并非所有操作都需要追 求 “细节”, 如在配制草酸标准溶液的操作中, 虽然在给定的实验方案中粗称的质量为 $1.58 \mathrm{~g}$, 但过分 追求 $1.58 \mathrm{~g}$ 显得毫无必要, 称量质量在 1.58 的 $\pm 10 \%$ 之内即可。

从授课者的角度来说, 可从以下四个方面入手, 让学生意识到这些问题关系到实验的质量。其 一, 化被动为主动, 让学生自己去思考哪些细节需要重点把握, 而非直接进行讲解传授, 如在讲解 邻二氮菲法测量碳酸锂样品中微量铁含量时, 可通过提问 (如 “哪些因素会影响到实验结果的准确 性” , 进而提出 “哪些操作会决定这些因素” )让学生意识到重点; 其二, 树立量和误差的概念, 有 实验的地方就有误差, 在误差允许范围内的实验结果都是可以接受的, 过度追求 “极致的准确” 并 不可取; 其三, 把握好实验进程, 这其中则需要耐心与细心, 对于学生过度追求准确的行为应该及 时指出, 同时在教授其不可行之时更应该强调为何不可行; 其四是及时做好反馈, 根据实验结果引 导学生进行反思。

\section{表1 实验中常见细节性错误(共性错误只列一次)}

\begin{tabular}{cl}
\hline 实验名称 & \multicolumn{1}{c}{ 错误描述 } \\
\hline 经典滴定与电位滴定 & 洗涤不够, 转移不充分 \\
& 定容后未充分摇匀, 摚拌不充分 \\
& 电位滴定时挂在烧杯内壁的溶液忘记清洗下去 \\
& 滴定间隔太大 \\
& 转移称量物时忘记变换把控称量瓶的手势 \\
& 以移液管移取溶液时伸入溶液过深 \\
& 以移液管移放溶液时尖端未靠壁 \\
& 润湿碳酸锂试样时加水过量 \\
& 定容时过标线 \\
& 滴定操作错误 \\
& 转移样品溶液时洗涤没有少量多次(导致加入水太多) \\
& 忘记加入关键试剂(如缓冲试剂或显色剂) \\
\hline
\end{tabular}


“细节决定成败” 固然有理, 但其必须建立在方向正确的基础之上, 如果方向出现错误, 过度 的处理细节也无济于事。

\section{4 习惯成自然一一树立良好的操作习惯}

仅仅通过记忆去记住繁琐复杂的实验操作对于初学者而言并非易事, 因此实验习惯的培养就显 得尤为重要, 如果能够将实验操作内化于心, 那么就很容易将庞大的实验内容构建出一张清晰的网 络 ${ }^{[4]}$ 。

吸收知识的初步阶段在于记忆和理解, 当逐步深化、归纳后, 良好的操作习惯和思维习惯也就 会逐渐形成。实验教学中一个经常遇到但又不可回避的问题是, 在教师传授过要点后学生自行操作 往往出现遗忘以及错误操作等, 对初学者尤其明显。这很大程度上是由于初学者难以接受繁琐的实 验要点, 而相关理论知识的缺乏使得其又面临分身乏术的困境, 最终的结果就是错误操作。如笔者 在讲授草酸标准溶液配制时, 在强调溶液摇匀的前提下依然会有少数学生实验过程中出现未充分摇 匀的情况。习惯成自然—这也是保持记忆的常用方法。

对于授课者而言, 至少需要做到以下四点: 其一, 实验演示必须有序且合理。实验演示对于学 生而言是一个很重要的学习途径, 如果教学者在实验演示部分操作凌乱, 很容易使得原本容易为学 生接受的内容变得复杂; 其二, 思考。引导学生去思考操作是否正确而非简单地告诉其操作流程。 如移液管或滴定管的润洗操作, 由于移液管移取溶液自下至上, 因此润洗时只需要将最后的溶液从 下口放掉, 而滴定管则不同, 外加溶液时从上往下加入, 因此润洗时部分溶液需要沿上口润洗放出。 分析道理固然简单, 但是却可以引导学生思考实验中的操作原理; 其三, 归纳。良好的知识整理和 归纳有利于教学的有序进行, 通过构造简易的 “口诀” 等可以将复杂的实验步骤简单化, 如重量分 析法中 “稀、酸、热、慢、摚、陈” 很轻易地将沉淀分析中几个关键点衔接了起来; 其四, 实验过 程中的监督与提醒, 及时地纠正错误远比 “扣分” 等更有助于帮助学生培养良好的习惯。图2显示的 是 “光度法” 和 “经典滴定与电位滴定” 实验中学生实验结果与操作之间的相关性(62份样本)。

培养良好的实验习惯同样不能忽略对细节的要求, 即使其未必会影响实验结果。除了如实记录 实验中的数据外, 影响实验结果的数据同样应该记录, 如以 $\mathrm{pH}$ 计测量 $\mathrm{pH}$ 以及以光度计测量吸光度的 实验中, 尽管温度这个量不会直接参与到最终的结果计算中, 但实际上温度对于实验结果同样会产 生一定的影响, 因此应该如实记录实验条件下的温度。养成良好的实验习惯有利于误差的分析、数 据的总结以及结论的判断。同样地, 绿色化学、环境保护、合作共赢等同时也应该渗透到实验教学 中的各个方面。

良好习惯的培养是一个相对漫长的过程, 但并非遥不可及, 毕竟对于定量分析实验而言, 许多 操作背后的道理都极其相似, 举一反三, 不断强调、深化, 形成一个良好的实验习惯也就水到渠成。
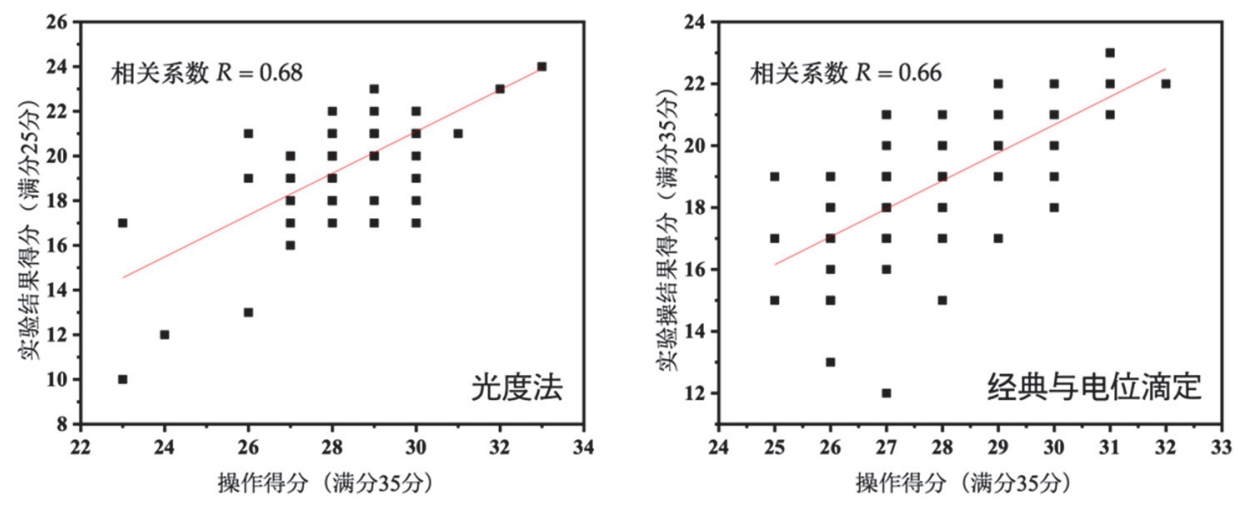

图2 实验结果与操作之间的相关性 


\section{5 上下同欲者胜一一合作}

实验教学中的合作主要体现在小组实验中, 为保证实验教学的质量与效率, 小组实验人数需要 控制在合理范围内。例如 “光度法” 实验, 按照正常的操作进行 (不出现较大失误), 两人一组基本能 够顺利、高质量完成实验。但实验结果的质量很大程度上取决于工作分配的合理与否。笔者发现大 部分学生合作基本能够做到有序进行, 而对于知识、能力相差较大的一组学生而言, 往往会出现并 不太 “和谐” 的一面, 如能力更强的学生可能会承担相对更多的实验任务, 一些优秀的学生甚至会 觉得自己被 “拖累”等。

良好的合作关系是高质量完成工作的基本保障, 实验教学中强调个人能力的提升, 但同时也需 要训练团队工作能力。笔者认为小组实验教学的主要任务在于构建良好的合作关系, 根据各自长处 合理进行相关的工作，勠力同心，教师更多承担的应该是引导作用。

\section{6 归纳与比较}

几乎所有的实验操作都可追溯至基本实验操作, 训练基本操作也自然成为了基础实验教学中的 一大任务。定量分析化学实验涉及到的主要是 “量” 的概念, 由此延伸出了一系列对于实验操作的 基本要求。实验内容不计其数, 基本实验操作却屈指可数, 对基本实验操作、实验内容、分析方法 进行归纳与比较往往可以获得事半功倍的效果。如几乎所有实验都涉及到了 “量” , 不同物质在分 析方法的选择上不尽相同, 如常量的钡离子可采用重量分析法进行分析, 而常量的铁则可以络合滴 定法进行分析; 当然, 不同量的相同物质在分析方法的选择上也有所差异, 如常量铁和微量铁的分 析方法就有很大不同。

及时归纳与总结有助于更全面、深刻地把握实验要点和知识点。在实验教学中, 帮助学生养成 归纳与比较的习惯将更有利于其在繁琐复杂的实验内容和操作中明晰原理、熟悉操作等内容。

\section{7 海纳百川一吸收}

教学从来都不是一个单向的行为, 教学相长, 作为一个相互学习的平台, 学生的反馈同样重要。

自然科学往往更强调逻辑性, 传授知识应该更加注重推理与逻辑, 定量分析化学实验也不例外。 实验教学同样应该建立在逻辑的基础之上, 而非一味地去告诉学生如何去做, 更多的应该是去告诉 他们原理以及思考的方向。作为学生, 在接受知识与经验的同时也是思考的过程, 许多学生的创造 性思维同样是实验中不可缺少的部分。作为教师, 应该对这些进行分析判断, 对于合理的提议应当 接纳、总结与传承。笔者在讲授 “邻二氮菲分光光度法测定微量铁含量” 时, 其中一个实验内容是 绘制吸光度-波长曲线, 讲解过程中很自然地强调注意加入试剂的顺序, 但也有两组学生提出想尝试 一下未按照指定顺序加入试剂的结果; 再如在探究 $\mathrm{pH}$ 对邻二氮菲-铁络合物稳定性的影响时, 尽管实 验是通过滴定管滴加 $\mathrm{NaOH}$, 但事实上绝大部分学生都没有获得 $\mathrm{pH}$ 在 8-11之间的梯度, 他们忽略了 这个实验细节, 仅有极少数同学通过分析发现其实这一段 $\mathrm{pH}$ 恰好处在滴定突跃的变化范围内, 显然 要获得突变范围内的 $\mathrm{pH}$ 是相对困难的。

教学中会遇到很多意料之外的事情, 许多未被思考的问题可能会被学生进一步提出(表2), 而如 何去反思、总结甚至接纳这些问题值得每一个授课者去思考。

\section{4 定量分析教学新模式思考}

\section{1 引入实际分析体系, 增加实验前处理}

相对于具备完善实验教案的模拟样品而言, 实际样品的分析中前处理和数据分析往往更加复杂。 以 “工业碳酸锂中微量铁的测定” 为例, 给定的方案是通过光度法进行测定, 但在缺乏相关含量、 组分信息的前提下, 实验的设计可能需要考虑到实验方案的选择、干扰物质的排除、样品的前处理 等过程。再以 “未知酸分子量的测定” 为例, 实际体系中涉及到的未知酸可能会涉及到是否为一元 酸或二元酸、能否分步滴定甚至能否滴定等一系列问题，更加具有挑战性。 
表2 实验中学生常提出的问题

\begin{tabular}{cl}
\hline 实验名称 & \multicolumn{1}{c}{ 问题 } \\
\hline 经典滴定与电位滴定 & 能否在电位滴定时加入指示剂? \\
& 为什么先要在千分之一天平上粗称后在在万分之一天平上称量草酸样品? \\
& 电位滴定时摚拌速率的问题以及什么时候需要摚拌? \\
& 二阶微分不可导怎么办? \\
& 处理试样溶液时为什么要将溶液蒸干? \\
& $\mathrm{pH}$ 探究实验时为什么需要加热? \\
& $\mathrm{pH}$ 光探究实验时有哪些 $\mathrm{pH}$ 很难达到? \\
& 为什么以试剂空白为参比? \\
& 为什么络合物的吸光度与波长有关? \\
\hline
\end{tabular}

与物理化学实验、有机化学实验不同的是, 定量分析化学实验可以更加贴近生活。选择定量分 析化学实验时可以引入实际样品分析, 组分不必复杂, 但应该涉及到定量分析化学实验中从样品采 集、前处理到质量控制的一整套定量分析过程。由此可以加深学生对于分析方法建立、样品前处理 等基本流程的熟悉与理解, 进而加强学生解决实际问题的能力。

\section{2 完善课程反馈与评估, 建立积极的交流关系}

学生与教师之间的关系不应该是孤立的, 良好的教学质量应该建立在学生与教师积极的交流关 系上。课堂是学生和助教共同的课堂, 在实验教学中, 助教应该加强实验课堂的巡视和指导, 及时 纠正学生实验过程中的不规范操作, 避免学生二次犯错。做好实验课程的反馈, 通过电子问卷等途 径及时获取学生对于实验课程的意见与反馈并且及时进行讨论和评估, 进一步完善学生-助教之间的 沟通渠道, 也有利于使助教明确学生的需求, 针对性给予一定的指导。对助教的评估也有助于及时 裨补缺漏, 提升教学能力。

从课堂上反馈来看, 很大一部分学生希望课后能有一些积极的交流或讨论, 部分学生能够积极 参与到课堂讨论中, 并提出了关于实验内容的一些想法和见解。学生能够积极思考是良好教学的一 种体现, 若助教能够积极去引导和解答, 必然会给学生带来更加积极的影响。通过反馈、评估与交 流, 学生、助教及主讲教师将由以往的梯次关系转变为积极的交流互动关系(图3)。

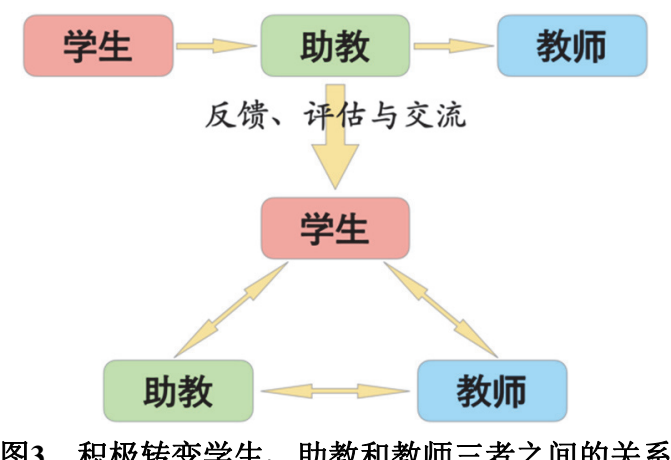

\section{3 规划自由实验时间, 加强学生探究能力}

当前实验教学中普遍通行的实施办法是在固定的时间和地点安排一定的教学任务, 教师与学生 各自完成教与学的任务即意味此次实验课的结束。这种模式基本能够满足绝大部分学生的需求, 但 少数学生可能会表现出更强的求知欲。有些学生对实验中的某部分内容较感兴趣, 希望进一步探究。 如光度法实验中, 部分学生对 “加入试剂的顺序对吸光度的影响” 较感兴趣; 在进行未知酸分子量 
的测定实验中, 部分学生希望通过更多的数据来对比经典滴定与电位滴定, 甚至有学生希望利用分 析方法来确定未知酸的分子结构……但以上思考很多都因课堂学时所限而未能系统性地完成; 部分 学生更是希望课后能够进一步锻炼实验操作技能。

实验课的教学应以提高学生的知识能力等为主要目标, 在统筹好时间、地点等前提下, 可为学 生提供必要的实验药品及场地, 满足学生的实验需求, 从而为学生知识、能力、创造力的提升提供 良好的平台 ${ }^{[5,6]}$ 。

\section{4 完善助教培训, 增加研讨时间}

“工欲善其事, 必先利其器”, 助教在实验教学中承担了相当重要的一部分工作, 作为介于教 师-学生之间的身份角色, 助教在与学生交流沟通方面具备一定的优势, 但是由于知识经验等方面的 不足和缺乏, 助教也面临着一定的挑战。欲提高教学质量, 助教相关能力的提高势在必行。

助教培训是助教从学生走向教师一个很重要的阶段, 合理构建实验教学体系、进一步规范化实 验操作、尽心指导学生实验、公平评价学生等都是助教培训中很重要的过程, 不可仅局限在实验操 作的规范化上。要把握好从理论讲解到实验评分的每一个环节, 建立起规范严格的助教培训体系。

安排助教研讨时间, 针对同一实验教学, 助教间应该加强沟通, 及时了解其他实验室学生的教 学情况, 以此避免信息不对称, 增强对学生知识能力掌握程度的了解。

增加学生与教师的研讨时间, 笔者在参与的几个实验中, 均有学生提出自己的思考, 这是一个 良好的开始, 笔者认为可以合理安排研讨时间, 进一步加强实验教学效果。

\section{5 鼓励学生自行提出实验, 增加个性化设计}

实验设计通常由教师主导, 因此学生的主观能动性有限, 而定量分析化学实验与生活联系紧密, 若由学生自行提出实验方案, 经过与教师进行讨论后再拟定, 不仅可以增强学生的思考能力, 而且 能够提高学生的主观能动性, 由此极大增强了其分析实际样品的能力。当然也需要建立一定的激励 机制来确保设计实验的质量。尽管该方案的实施对于大一学生来说具有一定的难度, 但是并非不切 实际。

\section{5 结语}

定量分析化学实验教学及改革是一个复杂的工程。目前的实验教学正在向立足基础教学、联系 生活实际、走向生产前沿的方向发展, 因此对于学生和教师的要求也越来越高, 综合化、网络化、 立体化的实验教学方案已经逐渐成熟, 教学质量的优化也意味着教师与学生需要投入更多的时间去 思考教与学, 笔者仅从参与的实验教学出发, 立足于一个青年助教的视野, 以教与学过程中的一些 思考与经历提出一些想法与建议。

\section{参 考 文 献}

[1] 李克安. 分析化学教程. 北京: 北京大学出版社, 2005.

[2] 北京大学化学与分子工程学院分析化学教学组. 基础分析化学实验. 第3版. 北京: 北京大学出版社, 2010.

[3] 王宏, 陈浩, 陈志飞, 吴康兵, 聂进. 大学化学, 2011, 26 (1), 35 .

[4] 宿艳, 吴硕, 崔丽钧, 肖光. 实验室科学, 2015, 18 (2), 232.

[5] 郝玉翠, 艾智, 孟丽军. 大学化学, 2012, 27 (4), 20.

[6] 李松栋, 周激, 吴跃焕, 张翠红, 张颖. 实验科学与技术, 2012, 10 (3), 90 . 